\title{
Supersymmetric $U(1)$ Gauge Field Theory With Massive Gauge Field
}

\author{
Ning $\mathrm{Wu}$ * \\ CCAST (World Lab), P.O.Box 8730, Beijing 100080, P.R.China \\ and \\ Division 1, Institute of High Energy Physics, P.O.Box 918-1, Beijing 100039, P.R.China
}

June 16, 2021

PACS Numbers: 11.30.Pb, 12.60.J, 11.15-q,

Keywords: supersymmetry, gauge symmetry, gauge field, mass

\begin{abstract}
[Abstract] A supersymmetric model with $U(1)$ gauge symmetry will be discussed in this paper. The model has strict $U(1)$ gauge symmetry and supersymmetry simultaneously. Besides, there is a massive $U(1)$ gauge field contained in the model.
\end{abstract}

According to the conventional notion, if a system has strict gauge symmetry, the mass of gauge field in the lagrangian must be zero [1 ]. With the development of the gauge theory, this notion has already been changed. In the gauge theory developed by $\mathrm{Wu}[2$ ], under the precondition that the system has strict gauge symmetry, the theory contains massive gauge bosons. In fact, this result holds not only in the usual quantum field theory, but also in the supersymmetric quantum field theory. In other words, we could construct a supersymmetric gauge field model with massive gauge bosons. Supersymmetric gauge field models have been extensively discussed in the literature [3-5 ]. Similar to the conventional gauge model, in all these supersymmetric gauge models, the masses of gauge fields is zero. In this paper, we will construct a gauge field model which has strict $U(1)$ gauge symmetry, supersymmetry and one massive gauge field.

*email:wuning@tofj1.ihep.ac.cn 
In supersymmetry, gauge field comes from vector superfield. Vector superfield is denoted as $\mathrm{V}(x, \theta, \bar{\theta})$, where $x$ is space-time coordinate, $\theta$ and $\bar{\theta}$ are coordinates of superspace. Vector superfield satisfies reality condition:

$$
\mathrm{V}(x, \theta, \bar{\theta})=\mathrm{V}^{\dagger}(x, \theta, \bar{\theta})
$$

The most general vector superfield contains six component fields. In supersymmetric gauge field theory, we always select Wess-Zumino gauge. In this case, a vector superfield only contains three component fields. It can be expanded as:

$$
\mathrm{V}(x, \theta, \bar{\theta})=\theta \sigma^{\mu} \bar{\theta} v_{\mu}(x)+(\theta \theta) \bar{\theta} \bar{\lambda}(x)+(\bar{\theta} \bar{\theta}) \theta \lambda(x)+(\theta \theta)(\bar{\theta} \bar{\theta}) D(x)
$$

where $v_{\mu}(x)$ is vector field, $\lambda(x)$ is spinor field which is usually called gaugino field. In this paper, all vector superfields are required to satisfy Wess-Zumino gauge.

In supersymmetry, matter fields are usually described by chiral superfields. There are two kinds of chiral superfields, one is left-handed chiral superfield and another is right-handed chiral superfield. They satisfy the following conditions

$$
\begin{aligned}
\bar{D}_{\dot{\alpha}} \Phi(x, \theta, \bar{\theta}) & =0 \\
D_{\alpha} \Phi^{\dagger}(x, \theta, \bar{\theta}) & =0,
\end{aligned}
$$

where $\Phi^{\dagger}(x, \theta, \bar{\theta})$ is hermitian conjugation of $\Phi(x, \theta, \bar{\theta}), D_{\alpha}$ and $\bar{D}_{\dot{\alpha}}$ are covariant derivatives.

In this model, we will introduce two vector superfields $\mathrm{V}_{1}(x, \theta, \bar{\theta})$ and $\mathrm{V}_{2}(x, \theta, \bar{\theta})$ , and two chiral superfields $\Phi_{1}(x, \theta, \bar{\theta})$ and $\Phi_{2}(x, \theta, \bar{\theta})$. Two vector superfields are in the Wess-Zumino gauge. They can be expanded as:

$$
\mathrm{V}_{i}(x, \theta, \bar{\theta})=\theta \sigma^{\mu} \bar{\theta} v_{i \mu}(x)+(\theta \theta) \bar{\theta} \bar{\lambda}_{i}(x)+(\bar{\theta} \bar{\theta}) \theta \lambda_{i}(x)+(\theta \theta)(\bar{\theta} \bar{\theta}) D_{i}(x), \quad(i=1,2) .
$$

The chiral superfields have the following component expressions:

$$
\Phi_{i}(x, \theta, \bar{\theta})=A_{i}(y)+\sqrt{2} \theta \psi_{i}(y)+(\theta \theta) F_{i}(y), \quad(i=1,2)
$$

where

$$
y^{\mu}=x^{\mu}+i \theta \sigma^{\mu} \bar{\theta} .
$$

In order to obtain the kinetic energy terms, let's define

$$
\begin{aligned}
& W_{i \alpha}=-\frac{1}{4}(\bar{D} \bar{D}) D_{\alpha} \mathrm{V}_{i}(x, \theta, \bar{\theta}) \\
& \bar{W}_{i \dot{\alpha}}=-\frac{1}{4}(D D) \bar{D}_{\dot{\alpha}} \mathrm{V}_{i}(x, \theta, \bar{\theta})
\end{aligned}
$$

The lagrangian of the model is

$$
\begin{aligned}
S= & \int d^{4} x d^{4} \theta\left\{\frac{1}{4}\left(W_{1}^{\alpha} W_{1 \alpha}+W_{2}^{\alpha} W_{2 \alpha}\right) \delta^{2}(\bar{\theta})+\frac{1}{4}\left(\bar{W}_{1 \dot{\alpha}} \bar{W}_{1}^{\dot{\alpha}}+\bar{W}_{2 \dot{\alpha}} \bar{W}_{2}^{\dot{\alpha}}\right) \delta^{2}(\theta)\right. \\
& +\Phi_{1}^{\dagger} e^{2 e V_{1}} \Phi_{1}+\Phi_{2}^{\dagger} e^{-2 e V_{1}} \Phi_{2}-m \Phi_{1} \Phi_{2} \delta^{2}(\bar{\theta})-m \Phi_{1}^{\dagger} \Phi_{2}^{\dagger} \delta^{2}(\theta) \\
& \left.-\mu^{2}\left(\cos \alpha V_{1}+\sin \alpha V_{2}\right)^{2}\right\} .
\end{aligned}
$$


The supersymmetric $U(1)$ gauge transformations are:

$$
\begin{gathered}
\Phi_{1}(x, \theta, \bar{\theta}) \longrightarrow e^{-i e \Lambda} \Phi_{1}(x, \theta, \bar{\theta}) \\
\Phi_{2}(x, \theta, \bar{\theta}) \longrightarrow e^{i e \Lambda} \Phi_{2}(x, \theta, \bar{\theta}) \\
V_{1}(x, \theta, \bar{\theta}) \longrightarrow V_{1}(x, \theta, \bar{\theta})+\frac{i}{2}\left(\Lambda-\Lambda^{\dagger}\right) \\
V_{2}(x, \theta, \bar{\theta}) \longrightarrow V_{2}(x, \theta, \bar{\theta})-\frac{i}{2 \operatorname{tg} \alpha}\left(\Lambda-\Lambda^{\dagger}\right),
\end{gathered}
$$

where $\Lambda$ satisfies the following constrains:

$$
\begin{aligned}
\bar{D}_{\dot{\alpha}} \Lambda(x, \theta, \bar{\theta}) & =0 \\
D_{\alpha} \Lambda^{\dagger}(x, \theta, \bar{\theta}) & =0 .
\end{aligned}
$$

Corresponding to the Wess-Zumino gauge of vector superfields, some component fields of $\Lambda$ should be zero. In other words, $\Lambda$ 's component expression is

$$
\Lambda(x, \theta, \bar{\theta})=i B(x)-\theta \sigma^{\mu} \bar{\theta} \partial_{\mu} B(x)+i(\theta \theta)(\bar{\theta} \bar{\theta}) \partial^{\mu} \partial_{\mu} B(x),
$$

where $B(x)$ is a real scalar field. It is easy to prove that the action defined by eq(10) is invariant under the above supersymmetric $U(1)$ gauge transformations. Meanwhile, we will carry out integration over superspace coordinates, which equivalent to selecting the highest order component of the lagrangian. It is known that the highest order component of a superfield is invariant under supersymmetric transformation. So, the action defined by eq(10) has supersymmetry.

Substituting component expressions into the action (10) and carrying out the integration over superspace coordinates, we will change the action into the following form:

$$
\begin{aligned}
S= & \int d^{4} x\left\{2 D_{1}^{2}(x)+2 D_{2}^{2}(x)-\frac{1}{4} v_{1 \mu \nu}(x) v_{1}^{\mu \nu}(x)-\frac{1}{4} v_{2 \mu \nu}(x) v_{2}^{\mu \nu}(x)\right. \\
& -i \lambda_{1}(x) \sigma^{\mu} \partial_{\mu} \bar{\lambda}_{1}(x)-i \lambda_{2}(x) \sigma^{\mu} \partial_{\mu} \bar{\lambda}_{2}(x) \\
& +\left|F_{1}(x)\right|^{2}+i\left(D_{\mu}^{\star} \bar{\psi}_{1}(x)\right) \bar{\sigma}^{\mu} \psi_{1}(x)+\left|D_{\mu} A_{1}(x)\right|^{2}+2 e D_{1}(x)\left|A_{1}(x)\right|^{2} \\
& -\sqrt{2} e\left(\bar{\lambda}_{1}(x) \bar{\psi}_{1}(x) A_{1}(x)+\lambda_{1}(x) \psi_{1}(x) A_{1}^{\star}(x)\right) \\
& +\left|F_{2}(x)\right|^{2}+i\left(D_{\mu} \bar{\psi}_{2}(x)\right) \bar{\sigma}^{\mu} \psi_{2}(x)+\left|D_{\mu}^{\star} A_{2}(x)\right|^{2}+2 e D_{1}(x)\left|A_{2}(x)\right|^{2} \\
& +\sqrt{2} e\left(\bar{\lambda}_{1}(x) \bar{\psi}_{2}(x) A_{2}(x)+\lambda_{1}(x) \psi_{2}(x) A_{2}^{\star}(x)\right) \\
& +m\left(\psi_{1}(x) \psi_{2}(x)-A_{1}(x) F_{2}(x)-A_{2}(x) F_{1}(x)\right) \\
& +m\left(\bar{\psi}_{1}(x) \bar{\psi}_{2}(x)-A_{1}^{\star}(x) F_{2}^{\star}(x)-A_{2}^{\star}(x) F_{1}^{\star}(x)\right) \\
& \left.-\frac{\mu^{2}}{2}\left(\cos \alpha v_{1 \mu}+\sin \alpha v_{2 \mu}\right)\left(\cos \alpha v_{1}^{\mu}+\sin \alpha v_{2}^{\mu}\right)\right\}
\end{aligned}
$$

where

$$
\begin{gathered}
v_{i \mu \nu}=\partial_{\mu} v_{i \nu}-\partial_{\nu} v_{i \mu} \quad(i=1,2) \\
D_{\mu}=\partial_{\mu}-i e v_{1 \mu} \quad, \quad D_{\mu}^{\star}=\partial_{\mu}+i e v_{1 \mu}
\end{gathered}
$$


Now, let's consider the on-shell form of the aciton. In eq.(18), $F_{1}(x), F_{2}(x), D_{1}(x)$ and $D_{2}(x)$ are auxiliary fields which could be eliminated by applying their equations of motion. The equations of motion of these four auxiliary fields are:

$$
\begin{gathered}
F_{1}^{\star}(x)=m A_{2}(x) \\
F_{2}^{\star}(x)=m A_{1}(x) \\
D_{1}(x)=-\frac{e}{2}\left(\left|A_{1}(x)\right|^{2}-\left|A_{2}(x)\right|^{2}\right) \\
D_{2}(x)=0 .
\end{gathered}
$$

Applying these four equations of motion, we could rewritten the action as:

$$
\begin{aligned}
S= & \int d^{4} x\left\{-\frac{1}{4} v_{1 \mu \nu}(x) v_{1}^{\mu \nu}(x)-\frac{1}{4} v_{2 \mu \nu}(x) v_{2}^{\mu \nu}(x)-i \lambda_{1}(x) \sigma^{\mu} \partial_{\mu} \bar{\lambda}_{1}(x)-i \lambda_{2}(x) \sigma^{\mu} \partial_{\mu} \bar{\lambda}_{2}(x)\right. \\
& +i\left(D_{\mu}^{\star} \bar{\psi}_{1}(x)\right) \bar{\sigma}^{\mu} \psi_{1}(x)+i\left(D_{\mu} \bar{\psi}_{2}(x)\right) \bar{\sigma}^{\mu} \psi_{2}(x)+\left|D_{\mu} A_{1}(x)\right|^{2}+\left|D_{\mu}^{\star} A_{2}(x)\right|^{2} \\
& -\sqrt{2} e\left(\bar{\lambda}_{1}(x)\left(\bar{\psi}_{1}(x) A_{1}(x)-\bar{\psi}_{2}(x) A_{2}(x)\right)+\lambda_{1}(x)\left(\psi_{1}(x) A_{1}^{\star}(x)-\psi_{2}(x) A_{2}^{\star}(x)\right)\right) \\
& +m\left(\psi_{1}(x) \psi_{2}(x)+\bar{\psi}_{1}(x) \bar{\psi}_{2}(x)\right)-m^{2}\left(\left|A_{1}(x)\right|^{2}+\left|A_{2}(x)\right|^{2}\right)-\frac{e^{2}}{2}\left(\left|A_{1}(x)\right|^{2}-\left|A_{2}(x)\right|^{2}\right)^{2} \\
& \left.-\frac{\mu^{2}}{2}\left(\cos \alpha v_{1 \mu}+\sin \alpha v_{2 \mu}\right)\left(\cos \alpha v_{1}^{\mu}+\sin \alpha v_{2}^{\mu}\right)\right\} .
\end{aligned}
$$

Now, we change the two-component notations into four-component notations.

Define

$$
\begin{aligned}
& \psi(x)=\left(\begin{array}{c}
\psi_{1 \alpha}(x) \\
\bar{\psi}_{2}^{\alpha}(x)
\end{array}\right) \quad, \quad \bar{\psi}(x)=\left(\psi_{2}^{\alpha}(x), \bar{\psi}_{1 \dot{\alpha}}(x)\right) \\
& \lambda_{1}(x)=\left(\begin{array}{c}
\lambda_{1 \alpha}(x) \\
\bar{\lambda}_{1}^{\alpha}(x)
\end{array}\right) \quad, \quad \bar{\lambda}_{1}(x)=\left(\lambda_{1}^{\alpha}(x), \bar{\lambda}_{1 \dot{\alpha}}(x)\right) \\
& \lambda_{2}(x)=\left(\begin{array}{c}
\lambda_{2 \alpha}(x) \\
\bar{\lambda}_{2}^{\alpha}(x)
\end{array}\right) \quad, \quad \bar{\lambda}_{2}(x)=\left(\lambda_{2}^{\alpha}(x), \bar{\lambda}_{2 \dot{\alpha}}(x)\right) .
\end{aligned}
$$

Then the action (25) will be changed into:

$$
\begin{aligned}
& S=\int d^{4} x\left\{-\frac{1}{4} v_{1 \mu \nu}(x) v_{1}^{\mu \nu}(x)-\frac{1}{4} v_{2 \mu \nu}(x) v_{2}^{\mu \nu}(x)-\frac{i}{2} \bar{\lambda}_{1}(x) \gamma^{\mu} \partial_{\mu} \lambda_{1}(x)-\frac{i}{2} \bar{\lambda}_{2}(x) \gamma^{\mu} \partial_{\mu} \lambda_{2}(x)\right. \\
& -\bar{\psi}\left(i \gamma^{\mu} D_{\mu}-m\right) \psi+\left|D_{\mu} A_{1}(x)\right|^{2}+\left|D_{\mu}^{\star} A_{2}(x)\right|^{2}-m^{2}\left(\left|A_{1}(x)\right|^{2}+\left|A_{2}(x)\right|^{2}\right) \\
& -\frac{e}{\sqrt{2}}\left(\bar{\psi}\left(1+\gamma^{5}\right) \lambda_{1}(x) A_{1}(x)-\bar{\psi}(x)\left(1-\gamma^{5}\right) \lambda_{1}(x) A_{2}^{\star}(x)\right. \\
& \left.+\bar{\lambda}_{1}(x)\left(1-\gamma^{5}\right) \psi(x) A_{1}^{\star}(x)-\bar{\lambda}_{1}(x)\left(1+\gamma^{5}\right) \psi(x) A_{2}(x)\right) \\
& \left.-\frac{e^{2}}{2}\left(\left|A_{1}(x)\right|^{2}-\left|A_{2}(x)\right|^{2}\right)^{2}-\frac{\mu^{2}}{2}\left(\cos \alpha v_{1 \mu}+\sin \alpha v_{2 \mu}\right)\left(\cos \alpha v_{1}^{\mu}+\sin \alpha v_{2}^{\mu}\right)\right\} \text {. }
\end{aligned}
$$

Please note that $v_{1 \mu}$ and $v_{2 \mu}$ are not eigenvectors of mass matrix, so we make the following transformations:

$$
\begin{gathered}
F_{\mu}=\cos \alpha v_{1 \mu}+\sin \alpha v_{2 \mu} \\
F_{2 \mu}=-\sin \alpha v_{1 \mu}+\cos \alpha v_{2 \mu} .
\end{gathered}
$$

Correspondingly, the gauge covariant derivative becomes

$$
D_{\mu}=\partial_{\mu}-i e \cos \alpha F_{\mu}+i e \sin \alpha F_{2 \mu} .
$$


After these changes, the on-shell action becomes:

$$
\begin{aligned}
S= & \int d^{4} x\left\{-\frac{1}{4} F_{\mu \nu}(x) F^{\mu \nu}(x)-\frac{\mu^{2}}{2} F_{\mu} F^{\mu}-\frac{1}{4} F_{2 \mu \nu}(x) F_{2}^{\mu \nu}(x)\right. \\
& -\frac{i}{2} \bar{\lambda}_{1}(x) \gamma^{\mu} \partial_{\mu} \lambda_{1}(x)-\frac{i}{2} \bar{\lambda}_{2}(x) \gamma^{\mu} \partial_{\mu} \lambda_{2}(x)-\bar{\psi}\left(i \gamma^{\mu} D_{\mu}-m\right) \psi \\
& +\left|D_{\mu} A_{1}(x)\right|^{2}+\left|D_{\mu}^{\star} A_{2}(x)\right|^{2}-m^{2}\left(\left|A_{1}(x)\right|^{2}+\left|A_{2}(x)\right|^{2}\right)-\frac{e^{2}}{2}\left(\left|A_{1}(x)\right|^{2}-\left|A_{2}(x)\right|^{2}\right)^{2} \\
& -\frac{e}{\sqrt{2}}\left(\bar{\psi}(x)\left(1+\gamma^{5}\right) \lambda_{1}(x) A_{1}(x)-\bar{\psi}(x)\left(1-\gamma^{5}\right) \lambda_{1}(x) A_{2}^{\star}(x)\right. \\
& \left.\left.+\bar{\lambda}_{1}(x)\left(1-\gamma^{5}\right) \psi(x) A_{1}^{\star}(x)-\bar{\lambda}_{1}(x)\left(1+\gamma^{5}\right) \psi(x) A_{2}(x)\right)\right\},
\end{aligned}
$$

where ,

$$
\begin{gathered}
F_{\mu \nu}=\partial_{\mu} F_{\nu}-\partial_{\nu} F_{\mu} \\
F_{2 \mu \nu}=\partial_{\mu} F_{2 \nu}-\partial_{\nu} F_{2 \mu} .
\end{gathered}
$$

From on-shell action (33), we know that there exist two gauge fields $F_{\mu}(x)$ and $F_{2 \mu}(x)$ in the theory. And $F_{\mu}(x)$ is massive gauge field whose mass is $\mu, F_{2 \mu}(x)$ is massless gauge field. Correspondingly, there are two massless gaugino fields $\lambda_{1}(x)$ and $\lambda_{2}(x)$ in the theory. The matter fields in the theory are one spinor field $\psi(x)$ and two complex scalar fields $A_{1}(x)$ and $A_{2}(x)$ whose masses are $m$.

In the above discussions, only vector superfield $\mathrm{V}_{1}(x, \theta, \bar{\theta})$ couples to matter fields, vector superfield $\mathrm{V}_{2}(x, \theta, \bar{\theta})$ does not couple to matter fields directly. In fact, we could let both two vector superfields couple to matter fields. In this case, the action should be defined as:

$$
\begin{aligned}
S= & \int d^{4} x d^{4} \theta\left\{\frac{1}{4}\left(W_{1}^{\alpha} W_{1 \alpha}+W_{2}^{\alpha} W_{2 \alpha}\right) \delta^{2}(\bar{\theta})+\frac{1}{4}\left(\bar{W}_{1 \dot{\alpha}} \bar{W}_{1}^{\dot{\alpha}}+\bar{W}_{2 \dot{\alpha}} \bar{W}_{2}^{\dot{\alpha}}\right) \delta^{2}(\theta)\right. \\
& +\Phi_{1}^{\dagger} e^{2 e\left(\cos ^{2} \phi V_{1}-\operatorname{tg} \alpha \sin ^{2} \phi V_{2}\right)} \Phi_{1}+\Phi_{2}^{\dagger} e^{-2 e\left(\cos ^{2} \phi V_{1}-\operatorname{tg} \alpha \sin ^{2} \phi V_{2}\right)} \Phi_{2} \\
& \left.-m \Phi_{1} \Phi_{2} \delta^{2}(\bar{\theta})-m \Phi_{1}^{\dagger} \Phi_{2}^{\dagger} \delta^{2}(\theta)-\mu^{2}\left(\cos \alpha V_{1}+\sin \alpha V_{2}\right)^{2}\right\} .
\end{aligned}
$$

The above action has both supersymmetry and $U(1)$ gauge symmetry. The corresponding on-shell action is

$$
\begin{aligned}
S= & \int d^{4} x\left\{-\frac{1}{4} F_{\mu \nu}(x) F^{\mu \nu}(x)-\frac{\mu^{2}}{2} F_{\mu} F^{\mu}-\frac{1}{4} F_{2 \mu \nu}(x) F_{2}^{\mu \nu}(x)\right. \\
& -\frac{i}{2} \bar{\lambda}_{1}(x) \gamma^{\mu} \partial_{\mu} \lambda_{1}(x)-\frac{i}{2} \bar{\lambda}_{2}(x) \gamma^{\mu} \partial_{\mu} \lambda_{2}(x)-\bar{\psi}\left(i \gamma^{\mu} D_{\mu}^{\prime}-m\right) \psi \\
& +\left|D_{\mu}^{\prime} A_{1}(x)\right|^{2}+\left|D_{\mu}^{\prime \star} A_{2}(x)\right|^{2}-m^{2}\left(\left|A_{1}(x)\right|^{2}+\left|A_{2}(x)\right|^{2}\right) \\
& -\frac{e^{2}}{2}\left(\cos ^{4} \phi+\operatorname{tg}^{2} \alpha \sin ^{4} \phi\right)\left(\left|A_{1}(x)\right|^{2}-\left|A_{2}(x)\right|^{2}\right)^{2} \\
& -\frac{e}{\sqrt{2}} \bar{\psi}(x)\left(1+\gamma^{5}\right)\left(\cos ^{2} \phi \lambda_{1}(x)-\operatorname{tg} \alpha \sin ^{2} \phi \lambda_{2}(x)\right) A_{1}(x) \\
& -\bar{\psi}(x)\left(1-\gamma^{5}\right)\left(\cos ^{2} \phi \lambda_{1}(x)-\operatorname{tg} \alpha \sin ^{2} \phi \lambda_{2}(x)\right) A_{2}^{\star}(x) \\
& +\left(\cos ^{2} \phi \bar{\lambda}_{1}(x)-\operatorname{tg} \alpha \sin ^{2} \phi \bar{\lambda}_{2}(x)\right)\left(1-\gamma^{5}\right) \psi(x) A_{1}^{\star}(x) \\
& \left.\left.-\left(\cos ^{2} \phi \bar{\lambda}_{1}(x)-\operatorname{tg} \alpha \sin ^{2} \phi \bar{\lambda}_{2}(x)\right)\left(1+\gamma^{5}\right) \psi(x) A_{2}(x)\right)\right\},
\end{aligned}
$$

where,

$$
D_{\mu}^{\prime}=\partial_{\mu}-i e \frac{\cos ^{2} \phi-\sin ^{2} \alpha}{\cos \alpha} F_{\mu}+i e \sin \alpha F_{2 \mu} .
$$

It is clearly seen from eq(39) that gaugino field $\lambda_{2}(x)$ couples to matter fields directly. The coupling between vector superfield $\mathrm{V}_{2}(x, \theta, \bar{\theta})$ and matter fields does not affect the 
masses of particles, only affects the dynamical behavior of the system.

From above discussions, we see that, after introducing another vector superfield, we could make one of the gauge field massive and keep another gauge field massless. But both gaugino fields are massless. So, in this model, the various components of the supermultiplet do not have the same masses. But, the various components of matter fields supermultiplet have the same masses.

\section{Reference:}

[1 ] C.N.Yang, R.L.Mills, Phys Rev 96 (1954) 191

[2 ] Ning Wu, Gauge Field Model With Massive Gauge Bosons, hep-ph/9802236

[3 ] J.Wess and B.Zumino, Nucl. Phys. B78 (1974) 1

[4] S.Ferrara and B.Zumino, Nucl.Phys. B79 (1974) 413

[5 ] A.Salam and J.Strathdee, Phys. Lett. 51B (1974) 353 